\title{
Matrix isolation FTIR spectroscopic and theoretical study of methyl lactate ${ }^{\text {ts }}$
}

\author{
Ana Borba ${ }^{\mathrm{a}}$, Andrea Gómez-Zavaglia ${ }^{\mathrm{a}, \mathrm{b}}$, Leszek Lapinski ${ }^{\mathrm{c}}$, R. Fausto ${ }^{\mathrm{a}, *}$ \\ ${ }^{a}$ Department of Chemistry, University of Coimbra, Coimbra P-3004-535, Portugal \\ ${ }^{\mathrm{b}}$ Facultad de Farmacia y Bioquímica, Universidad de Buenos Aires, Buenos Aires RA-1113, Argentina \\ ${ }^{\mathrm{c}}$ Institute of Physics, Polish Academy of Science, Warsaw PL-02-668, Poland
}

Received 28 January 2004; received in revised form 15 March 2004; accepted 24 March 2004

Available online 20 May 2004

\begin{abstract}
Methyl lactate $\left[\mathrm{CH}\left(\mathrm{CH}_{3}\right) \mathrm{OHC}(=\mathrm{O}) \mathrm{OCH}_{3}\right]$ conformational space was investigated by extensive DFT/B3LYP/6-311++G(d,p) and MP2/6$31++\mathrm{G}(\mathrm{d}, \mathrm{p})$ calculations and matrix-isolation FTIR spectroscopy in argon and xenon matrices. From the seven different conformers predicted by the calculations, two forms (the most stable conformers yielded by the calculations: $S s C$ and $G s k C$, where the letters refer to the conformations assumed by the $\mathrm{HOCC}, \mathrm{OCC}=\mathrm{O}$ and $\mathrm{O}=\mathrm{COC}$ dihedrals, respectively) were observed and characterized spectroscopically. Conformer interconversion pathways were also theoretically investigated. The low calculated barrier associated with the $G^{\prime} s k^{\prime} C \rightarrow G s k C$ conversion $\left(\sim 1 \mathrm{~kJ} \mathrm{~mol}^{-1}\right)$ explains the absence of the third most stable form $\left(G^{\prime} s k^{\prime} C\right)$ of methyl lactate in low-temperature matrices. Deposition of methyl lactate at different temperatures, together with the theoretical data, aided to the full assignment of the observed spectra. (C) 2004 Elsevier B.V. All rights reserved.
\end{abstract}

Keywords: Methyl lactate; Conformational analysis; Matrix isolation; FTIR spectroscopy; DFT(B3LYP) and MP2 calculations

\section{Introduction}

Carboxylic compounds substituted at the $\alpha$ position with hydroxyl group deserved much attention in the past due to their important medical and pharmaceutical applications [1-6]. From the point of view of basic chemical research, these compounds look also as very attractive and challenging systems, since the close proximity of their $\alpha$-hydroxyl and carboxylic groups gives rise to different possible intramolecular interactions in the isolated molecules and may also lead to a variety of intermolecular interactions in the condensed phases.

Simple $\alpha$-hydroxy carboxylic acids, in particular, have been extensively studied, both theoretically and experimentally [7-12]. On the other hand, their methyl esters have not been paid so much attention. However, the substitution of the $\mathrm{OH}$ acid group by the $\mathrm{OCH}_{3}$ ester group hinders the formation of some of the intramolecular hydrogen bonds, and this makes methyl $\alpha$-hydroxy carboxylates very con-

\footnotetext{
ts Supplementary data associated with this article can be found, in the online version, at doi:10.1016/j.vibspec.2004.03.004.

*Corresponding author. Tel.: +351-239-854483; fax: +351-239-827703.

E-mail address: rfausto@ci.uc.pt (R. Fausto).
}

venient compounds to undertake a more detailed study of the intramolecular hydrogen-bond interactions involving both the $\alpha$-hydroxyl group and the $-\mathrm{C}(=\mathrm{O}) \mathrm{O}$ fragment, without the additional complexity due to the presence of the acid hydrogen atom.

Among the methyl $\alpha$-hydroxy carboxylates, methyl glycolate (MGly) and methyl $\alpha$-hydroxy-isobutyrate (MHIb) have been the most studied species, having already been the subject of detailed structural, thermodynamic and vibrational analysis, both for the isolated molecule and in the condensed phases [13-18]. Matrix isolation infrared spectroscopy studies previously undertaken on these two compounds in our laboratory allowed the identification and characterization, in both cases, of two different conformers ${ }^{1}$ $S s C$ and $G s k C$, which were also shown to be present in the gaseous phase [13-15]. The $S s C$ conformer exhibits a planar heavy atom framework, with a relatively strong

\footnotetext{
${ }^{1}$ All molecules discussed in this paper obey to the general formula $\mathrm{CR}_{1} \mathrm{R}_{2} \mathrm{OH}-\mathrm{C}(=\mathrm{O}) \mathrm{OR}_{3}$, with $\mathrm{R}_{1}$ and $\mathrm{R}_{2}$ equal to $\mathrm{H}$ in MGly and $\mathrm{CH}_{3}$ in MHIb and $\mathrm{R}_{3}$ equal to $\mathrm{CH}_{3}$ in both compounds. In methyl lactate, $\mathrm{R}_{1}$ is equal to $\mathrm{H}$ and $\mathrm{R}_{2}$ and $\mathrm{R}_{3}$ equal to $\mathrm{CH}_{3}$. Conformations of these molecules are here named by a sequence of three letters which relate with the conformations ( $S, s$, syn; A,a, anti; G, gauche; sk, skew; $C$, s-cis; T, s-trans) assumed by the $\mathrm{H}-\mathrm{O}-\mathrm{C}-\mathrm{C}, \mathrm{O}-\mathrm{C}-\mathrm{C}=\mathrm{O}$ and $\mathrm{O}=\mathrm{C}-\mathrm{O}-\mathrm{R}_{3}$ dihedral angles.
} 
$\mathrm{OH} \cdots \mathrm{O}=$ intramolecular hydrogen bond, and was found to correspond to the ground conformational state in both MGly and MHIb. The second conformer, GskC, is characterized by a weaker intramolecular hydrogen bond of the type $\mathrm{OH} \cdots \mathrm{O}_{\text {ester }}$. It can be obtained from the most stable form by internal rotation around the $\mathrm{C}-\mathrm{C}$ bond and has an energy higher than this latter by 9.0 and $6.6 \mathrm{~kJ} \mathrm{~mol}^{-1}$, for MGly and MHIb, respectively (calculated HF/6-31G ${ }^{*}$ [13-15]).

Methyl lactate (MLac) differs from MGly by substitution of one of the $\alpha$-hydrogen atoms by a methyl group. The $\alpha$ carbon atom is a chiral center and monomeric MLac is then an optically active species, exhibiting two spectroscopically equivalent enantiomers $(R$ and $S$ ). Its parent molecule, lactic acid (LA), was recently studied in our laboratory by matrix isolation spectroscopy and theoretical methods (DFT/ B3LYP, MP2) [7]. The detailed investigation of the potential energy surface of the monomeric form of LA revealed that, in the gaseous phase, this molecule may exist in four significantly populated conformers: the most stable $S s C$ conformer, and forms $G s k C, G^{\prime} s k^{\prime} C$ and AaT. All these conformers exhibit an intramolecular H-bond that accounts to some extent for their stability. In the $S s C$ form, the H-bond is of the $\mathrm{OH}_{\text {alcohol }} \cdots \mathrm{O}=$ type, whereas in both $G s k C$ and $G^{\prime} s k^{\prime} C$ it is of the $\mathrm{OH}_{\text {alcohol }} \cdots \mathrm{O}_{\text {acid }}$ type; in $A a T$, the $\mathrm{OH}$ acid group acts as $\mathrm{H}$-bond donor to the $\alpha$-oxygen atom $\left(\mathrm{OH}_{\text {acid }} \cdots \mathrm{O}_{\text {alcohol }}\right)$. The second $(G s k C)$ and third $\left(G^{\prime} s k^{\prime} C\right)$ most stable conformers differ from $\mathrm{Ss} C$ in the $\mathrm{CCOH}_{(\text {alcohol })}$ and $\mathrm{O}=\mathrm{CCO}$ dihedral angles, which are equal to $43.3^{\circ}$ and $156.8^{\circ}$, and $-51.5^{\circ}$ and $-149.9^{\circ}$, respectively in $G s k C$ and $G^{\prime} s k^{\prime} C$ [7]. Matrix isolation experiments confirmed the predominance of $S s C$ conformer in both argon and xenon matrices, and provided the first experimental observation of conformers GskC and AaT. On the other hand, since the energy barrier to convert the $G^{\prime} s k^{\prime} C$ conformer into the $G s k C$ is only $\sim 2 \mathrm{~kJ} \mathrm{~mol}^{-1}$, conformational cooling $[19,20]$ precludes the observation of the $G^{\prime} s k^{\prime} C$ form in low-temperature matrices. This last form converts easily to the slightly more stable GskC conformer [7].

Taking into consideration the results described above obtained for the analogous compounds, LA, MGly and MHIb, it could be expected that for MLac a successful experimental observation of forms $S s C$ and $G s k C$ (or $G s k C$ and $G^{\prime} s k^{\prime} C$ ) could be done easily. However, with a single exception, previous experimental studies on this molecule were only able to detect the expectedly most stable $S s C$ conformer [21-23]. Experimental evidence of the higher energy forms of MLac was only reported hitherto by Freedman, Nafie and coworkers [24,25], who have studied this compound in $\mathrm{CCl}_{4}$ solution by vibrational circular dichroism (VCD). These authors examined the $\mathrm{C}-\mathrm{H}$ and $\mathrm{OH}$ stretching spectral regions of the VCD spectrum of MLac and, by comparison with the corresponding calculated spectra obtained at the DFT(B3LYP)/6-31G(d) level of theory, were able to identify in the studied regions spectral features that can be ascribable to the major $S s C$ form and to the two minor conformers having the
$\mathrm{OH} \cdots \mathrm{O}_{\text {ester }}$ intramolecular H-bond (GskC and $G^{\prime} s k^{\prime} C$ ) $[24,25]$. To the best of our knowledge no other studies on the conformational preferences and vibrational properties of monomeric MLac have been reported hitherto. The gas phase self-aggregation behavior of MLac in supersonic jet expansions were recently studied by Borho and Suhm $[18,26]$ using the ragout-jet FTIR technique [27].

In this work, the potential energy surface of MLac was reinvestigated at a higher level of theory than previously reported [24] (both the DFT(B3LYP)/6-311++G(d,p) and MP2/6-31++G(d,p) levels of theory were used). The structural, energetic and vibrational data obtained theoretically was then used to help interpretation of the matrix isolation FTIR spectra obtained for the compound isolated in solid argon and xenon, enabling the full vibrational characterization of the most stable forms of the studied molecule.

\section{Materials and methods}

\subsection{Computational methodology}

The quantum chemical calculations were performed with Gaussian 98 [28] at the DFT and MP2 levels of theory, using the $6-311++\mathrm{G}(\mathrm{d}, \mathrm{p})$ and $6-31++\mathrm{G}(\mathrm{d}, \mathrm{p})$ basis sets, respectively [29]. The calculations were carried out with the threeparameter density functional abbreviated as B3LYP, which includes Becke's gradient exchange correction [30] the Lee et al. correlation functional [31] and the Vosko et al. correlation functional [32].

Conformations were optimized at each level of theory using the geometry direct inversion of the invariant subspace (GDIIS) method [33]. Vibrational frequencies were calculated at each level of theory and the nature of the critical points on the potential energy surface resulting from optimization was determined by inspection of the corresponding calculated Hessian matrix. All optimized structures were confirmed to be minimum energy conformations. The calculated frequencies were scaled down by a single factor $(0.978)[7,20]$ to correct them for the effects of basis set limitations, neglected part of electron correlation and anharmonicity effects, and used to assist the analysis of the experimental spectra and to account for the zero-point vibrational energy contribution to the total energy. Normal coordinate analysis was undertaken in the internal coordinates space as described by Schachtschneider [34] using the program BALGA and the optimized geometries and harmonic force constants resulting from the DFT(B3LYP)/ $6-311++\mathrm{G}(\mathrm{d}, \mathrm{p})$ calculations. Potential energy profiles for internal rotation were calculated performing a relaxed scan on the potential energy surface (PES) along the relevant reaction coordinates and the transition state structures for conformational interconversion obtained using the synchronous transit-guided quasi-Newton (STQN) method [35]. 


\subsection{Infrared spectroscopy}

$(S)$ - and $(R)$-MLac of spectral purity were obtained from Aldrich. The IR spectra were collected with $0.5 \mathrm{~cm}^{-1}$ spectral resolution on a Mattson (Infinity 60AR Series) Fourier transform infrared spectrometer equipped with a deuterated triglycine sulfate (DTGS) detector and $\mathrm{Ge} / \mathrm{KBr}$ beamsplitter.

In the matrix isolation experiments, a glass vacuum system and standard manometric procedures were used to deposit the matrix gas (argon N60; xenon N45, obtained from Air Liquide). Matrices were prepared by co-deposition, onto the cooled CsI substrate of the cryostat, of the matrix gas (Ar or $\mathrm{Xe}$ ) and MLac ( $S, R$ or the 1:1 racemic mixture) placed in a specially designed doubly thermostatable Knudsen cell with shut-off possibility whose main component is a NUPRO SS-4BMRG needle valve. The temperature of the cell could be controlled separately in the valve nozzle and the sample compartment, enabling a more precise control of the saturated gas pressure over the liquid compound and a better metering function of the valve. Further details of the experimental set up can be found in [36]. All experiments were done using an APD Cryogenics close-cycle helium refrigeration system with a DE-202A expander. During deposition of matrices the temperature of the CsI substrate was 9 or $20 \mathrm{~K}$. Necessary modifications of the sample compartment of the spectrometer were made in order to accommodate the cryostat head and allow efficient purging of the instrument by a stream of air from which both water and $\mathrm{CO}_{2}$ vapors were previously removed.

\section{Results and discussion}

\subsection{Geometries and energies}

MLac has three different internal rotation axes (defined by the $\mathrm{CCOH}, \mathrm{OCC}=\mathrm{O}$ and $\mathrm{O}=\mathrm{COC}$ dihedral angles) that can give rise to conformational isomers. All possible conformers belong to the $\mathrm{C}_{1}$ symmetry point group. As far as vibrational spectroscopy neglecting dichroic effects is concerned, chirality of methyl lactate does not represent a source of complication, because the spectra of both enantiomers are identical.

In this work, a full conformational search on both the DFT(B3LYP)/6-311++G(d,p) and MP2/6-31++G(d,p) potential energy surfaces of MLac was undertaken. Table 1 presents the calculated relative energies for the possible conformers of MLac, which are depicted in Fig. 1. Equilibrium geometries are given in Table 2.

In consonance with the literature data [21-26], the theoretical calculations predict conformer $S s C$ as being the conformational ground state. As for the parent molecule, lactic acid [7], the heavy atom framework of this conformer was found to be slightly deviated from planarity $(\mathrm{CCOH}$, $\mathrm{OCC}=\mathrm{O}$ and $\mathrm{O}=\mathrm{COC}$ dihedral angles were calculated at the
Table 1

Relative energies, including zero point vibrational contributions ${ }^{\mathrm{b}}$ for the conformers of MLac ${ }^{\mathrm{a}}$

\begin{tabular}{llll}
\hline Conformer & $\begin{array}{l}\text { DFT(B3LYP)/ } \\
6-311++\mathrm{G}(\mathrm{d}, \mathrm{p})\end{array}$ & $\begin{array}{l}\text { MP2/6-31++G } \\
(\mathrm{d}, \mathrm{p})\end{array}$ & $\begin{array}{l}\text { Population } \\
\text { (at 298 K) (\%) }\end{array}$ \\
\hline$S s C$ & $0.0(-1005353.37)^{\mathrm{c}}$ & $0.0(-1002258.49)^{\mathrm{c}}$ & 91.8 \\
$G s k C$ & 7.42 & 5.72 & 4.6 \\
$G^{\prime} s k^{\prime} C$ & 8.06 & 6.23 & 3.5 \\
$A s C$ & 20.06 & 18.76 & 0.0 \\
$A a C$ & 20.43 & 19.28 & 0.0 \\
$A s T$ & 37.39 & 38.03 & 0.0 \\
$A s k T$ & 48.87 & 48.98 & 0.0 \\
\hline
\end{tabular}

${ }^{\mathrm{a}}$ Energies in $\mathrm{kJ} \mathrm{mol}^{-1}$; conformers are depicted in Fig. 1.

b Zero-point energy corrections taken from DFT(B3LYP)/6$311++\mathrm{G}(\mathrm{d}, \mathrm{p})$ calculations

${ }^{c}$ Total energies with zero point vibrational energy contribution (Hartrees).

B3LYP level of theory as $2.9^{\circ},-4.6^{\circ}$ and $-0.2^{\circ}$, respectively). As already mentioned, this form is similar to the most stable conformer of the other simple $\alpha$-hydroxy substituted acids and methyl esters (lactic acid, and glycolic and $\alpha$-hydroxy isobutyric acids and their methyl esters [12-15]), being stabilized by an intramolecular H-bond established between the $\mathrm{OH}$ group and the carbonyl oxygen atom.

The second most stable conformer (GskC, in Fig. 1) is predicted to have $\mathrm{CCOH}, \mathrm{OCC}=\mathrm{O}$ and $\mathrm{O}=\mathrm{COC}$ dihedral angles equal to $-36.9^{\circ},-160.1^{\circ}$ and $0.5^{\circ}$, respectively (B3LYP values), while in the third most stable conformer $\left(G^{\prime} s k^{\prime} C\right.$ in Fig. 1) these dihedral angles are $44.3^{\circ}, 152.8^{\circ}$ and $-0.7^{\circ}$, respectively. These two forms are characterized by a weak $\mathrm{OH} \cdots \mathrm{O}$ intramolecular $\mathrm{H}$-bond and their relative energies, with respect to the conformational ground state, are predicted to be 7.42 and $8.06 \mathrm{~kJ} \mathrm{~mol}^{-1}$ (MP2: 5.72 and $6.23 \mathrm{~kJ} \mathrm{~mol}^{-1}$ ) (see Table 1).

In lactic acid, the fourth more stable conformer is $A a T$ (with a relative energy within a range of $10 \mathrm{~kJ} \mathrm{~mol}^{-1}$ [7]), which has no equivalent form in MLac since in this latter molecule the stabilizing $\mathrm{OH}_{\text {acid }} \cdots \mathrm{O}_{\text {alcohol }}$ hydrogen bond cannot operate due to the substitution of the carboxylic hydrogen atom by the methyl ester group. Indeed, in the ester, the close proximity of the methyl ester group and the hydroxyl oxygen atom leads to a strong repulsion that is reflected in the fact that the AaT conformation is not a minimum energy species. A minimum in the potential energy surface is located in the vicinity of the $A a T$ conformation, corresponding to the highest energy conformer of MLac, the $A s k T$ form (Fig. 1) with a DFT calculated relative energy higher than $48 \mathrm{~kJ} \mathrm{~mol}^{-1}$. In MLac, the fourth and fifth most stable conformers predicted theoretically are the $A a C$ and $A s C$ forms (Fig. 1), respectively, which are predicted to have nearly equal relative energies of ca. $20 \mathrm{~kJ} \mathrm{~mol}^{-1}$. In both cases, the Anti conformation adopted by the $\mathrm{CCOH}$ axis inhibits intramolecular H-bonding, thus making these conformers much less stable than the three low energy forms discussed above ( $S s C, G s k C$ and $G^{\prime} s k^{\prime} C$ ). Besides $A a C, A s C$ and $A s k T$, another high energy conformer 
Table 2

Optimized geometries for the conformers of MLac ${ }^{\mathrm{a}}$

\begin{tabular}{|c|c|c|c|c|c|c|c|c|c|c|c|c|c|c|}
\hline \multirow[t]{2}{*}{ Parameter } & \multicolumn{2}{|l|}{ SsC } & \multicolumn{2}{|l|}{ GskC } & \multicolumn{2}{|l|}{$G^{\prime} s k^{\prime} C$} & \multicolumn{2}{|l|}{$A s C$} & \multicolumn{2}{|l|}{$A a C$} & \multicolumn{2}{|l|}{$A s T$} & \multicolumn{2}{|l|}{ AskT } \\
\hline & B3LYP & MP2 & B3LYP & MP2 & B3LYP & MP2 & B3LYP & MP2 & B3LYP & MP2 & B3LYP & MP2 & B3LYP & MP2 \\
\hline \multicolumn{15}{|l|}{ Bond length (pm) } \\
\hline $\mathrm{C}_{2}-\mathrm{O}_{1}$ & 144.4 & 144.8 & 144.2 & 144.6 & 144.2 & 144.6 & 144.0 & 144.5 & 144.0 & 143.0 & 143.0 & 144.2 & 143.9 & 144.4 \\
\hline $\mathrm{O}_{1}-\mathrm{C}_{6}$ & 133.8 & 134.6 & 135.1 & 135.8 & 135.0 & 136.8 & 135.3 & 136.0 & 133.7 & 134.6 & 134.0 & 135.0 & 134.9 & 135.0 \\
\hline $\mathrm{O}_{14}-\mathrm{H}_{15}$ & 96.8 & 97.2 & 96.4 & 96.8 & 96.4 & 96.8 & 96.2 & 96.7 & 96.3 & 96.7 & 96.0 & 97.0 & 96.3 & 96.0 \\
\hline $\mathrm{C}_{6}=\mathrm{O}_{7}$ & 121.0 & 122.6 & 120.5 & 122.1 & 120.5 & 122.2 & 120.0 & 121.8 & 120.9 & 122.5 & 120.0 & 122.0 & 120.2 & 121.0 \\
\hline $\mathrm{C}_{6}-\mathrm{C}_{8}$ & 152.5 & 151.8 & 153.1 & 152.4 & 152.8 & 151.9 & 152.7 & 151.8 & 152.6 & 151.7 & 153.0 & 152.0 & 154.1 & 153.0 \\
\hline $\mathrm{C}_{2}-\mathrm{H}_{3}$ & 108.7 & 108.4 & 108.7 & 108.4 & 108.7 & 108.4 & 108.8 & 108.4 & 108.7 & 107.0 & 108.0 & 108.0 & 109.0 & 108.0 \\
\hline $\mathrm{C}_{2}-\mathrm{H}_{4}$ & 109.0 & 108.7 & 109.0 & 108.7 & 109.0 & 108.7 & 109.1 & 108.8 & 109.1 & 107.0 & 109.0 & 108.0 & 108.0 & 108.0 \\
\hline $\mathrm{C}_{2}-\mathrm{H}_{5}$ & 109.0 & 108.7 & 109.0 & 108.7 & 109.0 & 108.7 & 109.1 & 108.7 & 109.1 & 107.0 & 109.0 & 108.0 & 108.0 & 108.0 \\
\hline $\mathrm{C}_{8}-\mathrm{H}_{9}$ & 110.0 & 109.5 & 110.3 & 109.9 & 109.2 & 108.9 & 109.7 & 109.4 & 110.0 & 109.3 & 109.0 & 108.0 & 110.0 & 109.0 \\
\hline $\mathrm{C}_{8}-\mathrm{C}_{10}$ & 153.2 & 152.4 & 152.2 & 151.5 & 153.5 & 152.7 & 153.4 & 152.6 & 153.3 & 152.5 & 153.0 & 152.0 & 152.0 & 151.0 \\
\hline $\mathrm{C}_{8}-\mathrm{O}_{14}$ & 141.4 & 142.0 & 142.0 & 142.5 & 141.9 & 142.4 & 141.8 & 142.4 & 142.1 & 142.8 & 141.0 & 141.0 & 143.0 & 143.0 \\
\hline $\mathrm{C}_{10}-\mathrm{H}_{11}$ & 109.3 & 109.0 & 109.2 & 108.9 & 109.4 & 109.1 & 109.2 & 109.0 & 109.2 & 109.0 & 109.0 & 108.0 & 109.0 & 109.0 \\
\hline $\mathrm{C}_{10}-\mathrm{H}_{12}$ & 109.2 & 108.9 & 109.0 & 108.8 & 109.3 & 109.0 & 109.2 & 109.0 & 109.2 & 109.0 & 109.0 & 108.0 & 109.0 & 108.0 \\
\hline $\mathrm{C}_{10}-\mathrm{H}_{13}$ & 109.1 & 108.8 & 109.1 & 108.8 & 109.1 & 108.9 & 109.4 & 109.1 & 109.4 & 109.1 & 109.0 & 109.0 & 108.0 & 108.0 \\
\hline \multicolumn{15}{|l|}{ Bond angles $\left({ }^{\circ}\right)$} \\
\hline $\mathrm{C}_{2}-\mathrm{O}_{1}-\mathrm{C}_{6}$ & 116.3 & 114.7 & 116.3 & 114.6 & 116.3 & 114.7 & 115.9 & 114.3 & 115.9 & 114.4 & 122.0 & 120.1 & 123.1 & 121.0 \\
\hline $\mathrm{O}_{1}-\mathrm{C}_{2}-\mathrm{H}_{3}$ & 105.4 & 105.0 & 105.5 & 105.0 & 105.5 & 105.0 & 105.5 & 105.0 & 105.3 & 109.5 & 111.9 & 7 & 104.9 & 104.5 \\
\hline $\mathrm{O}_{1}-\mathrm{C}_{2}-\mathrm{H}_{4}$ & 110.3 & 110.0 & 110.2 & 110.0 & 110.1 & 110.0 & 110.5 & 110.1 & 110.3 & 109.5 & 111.0 & 110.7 & 111.1 & 111.1 \\
\hline $\mathrm{O}_{1}-\mathrm{C}_{2}-\mathrm{H}_{5}$ & 110.2 & 110.0 & 110.2 & 110.0 & 110.3 & 110.0 & 110.3 & 110.1 & 110.5 & 109.5 & 105.1 & 104.6 & 111.0 & 110.4 \\
\hline $\mathrm{H}_{3}-\mathrm{C}_{2}-\mathrm{H}_{4}$ & 10.7 & 111.1 & 110.8 & 111.1 & 110.7 & 111.1 & 110.7 & 111.0 & 110.7 & 109.5 & 110.2 & 110.6 & 110.2 & 110.4 \\
\hline $\mathrm{H}_{3}-\mathrm{C}_{2}-\mathrm{H}_{5}$ & 0.8 & 111.1 & 110.7 & 111.1 & 110.8 & 111.1 & 110.6 & 111.0 & 110.8 & 109.5 & 109.2 & & 110.6 & 109.3 \\
\hline $\mathrm{H}_{4}-\mathrm{C}_{2}-\mathrm{H}_{5}$ & 109.4 & 110.0 & 109.4 & 109.7 & 109.4 & 109.7 & 109.2 & 109.5 & 109.2 & 109.5 & 109.3 & .7 & 10 & 111.0 \\
\hline $\mathrm{O}_{1}-\mathrm{C}_{6}=\mathrm{O}_{7}$ & 124.3 & 124.5 & 123.6 & 123.7 & 123.9 & 124.0 & 124.1 & 124.3 & 124.3 & 124.5 & 119.4 & 119.6 & 119.2 & 119.4 \\
\hline $\mathrm{O}_{1}-\mathrm{C}_{6}-\mathrm{C}_{8}$ & 112.6 & 112.1 & 111.3 & 111.1 & 111.4 & 110.9 & 109.6 & 109.4 & 113.3 & 112.2 & 120.2 & 119.9 & 118.5 & 118.2 \\
\hline $\mathrm{O}_{7}=\mathrm{C}_{6}-\mathrm{C}_{8}$ & 123.1 & 123.5 & 125.0 & 125.1 & 124.6 & 124.9 & 126.3 & 126.2 & 122.3 & 123.3 & 120.3 & 120.4 & 122.4 & 122.4 \\
\hline $\mathrm{C}_{6}-\mathrm{C}_{8}-\mathrm{C}_{10}$ & 111.9 & 111.3 & 111.7 & 111.4 & 110.0 & 109.4 & 110.0 & 109.3 & 110.0 & 109.8 & 109.9 & 109.2 & 111.4 & 111.2 \\
\hline $\mathrm{C}_{6}-\mathrm{C}_{8}-\mathrm{H}_{9}$ & 106.9 & 107.4 & 104.5 & 105.6 & 106.2 & 106.8 & 107.2 & 107.7 & 105.0 & 105.7 & 110.6 & 111.2 & 107.8 & 108.9 \\
\hline $\mathrm{C}_{6}-\mathrm{C}_{8}-\mathrm{O}_{14}$ & 109.4 & 109.5 & 113.2 & 112.4 & 113.1 & 112.4 & 107.2 & 106.6 & 109.7 & 108.0 & 108.9 & 108.9 & 106.3 & 105.1 \\
\hline $\mathrm{H}_{9}-\mathrm{C}_{8}-\mathrm{C}_{10}$ & 90 & 6 & 9 & 109.7 & 109.4 & .1 & 109.4 & 110.1 & 109.3 & .8 & 109.7 & .4 & .4 & 109.7 \\
\hline $\mathrm{H}_{9}-\mathrm{C}_{8}-\mathrm{O}_{14}$ & 9.0 & 10 & 10 & 11 & 109.1 & .8 & 110.7 & 110.9 & 110.6 & 11 & 10 & 106.2 & 109.4 & 109.6 \\
\hline $\mathrm{C}_{10}-\mathrm{C}_{8}-\mathrm{O}_{14}$ & 110.6 & 110.0 & 108.5 & 107.7 & 112.2 & 112.2 & 112.4 & 112.1 & 112.4 & 109.7 & 111.0 & 111.0 & 112.4 & 112.2 \\
\hline $\mathrm{C}_{8}-\mathrm{C}_{10}-\mathrm{H}_{11}$ & 110.5 & 109.9 & 110.3 & 110.0 & 110.8 & 110.2 & 110.2 & 109.5 & 110.4 & 110.5 & 109.9 & 109.3 & 110.6 & 110.1 \\
\hline $\mathrm{C}_{8}-\mathrm{C}_{10}-\mathrm{H}_{12}$ & 111.0 & 110.9 & 110.5 & 110.4 & 110.8 & 110.6 & 110.9 & 110.6 & 110.7 & 110.5 & 112.5 & 112.2 & 110.9 & 110.5 \\
\hline $\mathrm{C}_{8}-\mathrm{C}_{10}-\mathrm{H}_{13}$ & 108.8 & 108.7 & 109.2 & 109.0 & 109.0 & 109.0 & 110.0 & 110.0 & 109.9 & 109.9 & 10 & .8 & 7 & 109.6 \\
\hline $\mathrm{H}_{11}-\mathrm{C}_{10}-\mathrm{H}_{12}$ & 109.3 & 109.6 & 109.0 & 109.1 & 108.7 & 109.0 & 109.3 & 109.6 & 109.3 & 109.6 & 108.5 & 108.7 & 108.2 & 108.6 \\
\hline $\mathrm{H}_{11}-\mathrm{C}_{10}-\mathrm{H}_{13}$ & 108.2 & 108.4 & 108.5 & 108.9 & 108.4 & 108.8 & 108.6 & 108.8 & 108.6 & 109.0 & 108.5 & 108.8 & 109.3 & 109.8 \\
\hline $\mathrm{H}_{12}-\mathrm{C}_{10}-\mathrm{H}_{13}$ & 109.0 & 109.3 & 109.3 & 109.6 & 109.0 & 109.2 & 107.9 & 108.2 & 107.9 & 108.1 & 108.6 & 109.1 & 108.2 & 108.4 \\
\hline $\mathrm{C}_{8}-\mathrm{O}_{14}-\mathrm{H}_{15}$ & 07.2 & 106.8 & 108.5 & 108.0 & 108.3 & 107.6 & 108.3 & 107.6 & 108.3 & 107.6 & 106.7 & 106.1 & 108.4 & 107.7 \\
\hline \multicolumn{15}{|l|}{ Dihedral angles } \\
\hline $\mathrm{C}_{6}-\mathrm{O}_{1}-\mathrm{C}_{2}-\mathrm{H}_{3}$ & 179.6 & 9.4 & 180.0 & 180.0 & 179.5 & 179.6 & -179.7 & -179.4 & -178.8 & -47.7 & 62.5 & 61.5 & -64.0 & -54.6 \\
\hline $\mathrm{C}_{6}-\mathrm{O}_{1}-\mathrm{C}_{2}-\mathrm{H}_{4}$ & -60.9 & -61.1 & -60.4 & -60.4 & -60.0 & -60.1 & -60.0 & -59.8 & -59.3 & 72.3 & -61.0 & -62.2 & 58.9 & 69.0 \\
\hline $\mathrm{C}_{6}-\mathrm{O}_{1}-\mathrm{C}_{2}-\mathrm{H}_{5}$ & 60.0 & 60.0 & 60.4 & 60.5 & 60.8 & 60.7 & 60.8 & 61.1 & 61.5 & -167.7 & -179.0 & 179.8 & 176.6 & -173.6 \\
\hline $\mathrm{C}_{2}-\mathrm{O}_{1}-\mathrm{C}_{6}=\mathrm{O}_{7}$ & -0.2 & 0.0 & 0.5 & 0.6 & -0.7 & -0.7 & -1.3 & -1.5 & -1.4 & -1.8 & 169.6 & 169.2 & -175.9 & -174.2 \\
\hline $\mathrm{C}_{2}-\mathrm{O}_{1}-\mathrm{C}_{6}-\mathrm{C}_{8}$ & -179.9 & 179.7 & 177.6 & 177.4 & -178.2 & -177.3 & 176.9 & 175.7 & -178.8 & -178.3 & -13.4 & -15.4 & 3.2 & 6.5 \\
\hline $\mathrm{O}_{1}-\mathrm{C}_{6}-\mathrm{C}_{8}-\mathrm{C}_{10}$ & -62.0 & -62.4 & 145.8 & 150.3 & 96.3 & 89.6 & 80.8 & -85.4 & 94.2 & 80.1 & -80.3 & -81.6 & -175.8 & -172.7 \\
\hline $\mathrm{O}_{1}-\mathrm{C}_{6}-\mathrm{C}_{8}-\mathrm{H}_{9}$ & 57.3 & 57.6 & -96.6 & -90.7 & -145.8 & -151.3 & 37.9 & 34.2 & -148.5 & -161.0 & 41.0 & 40.4 & -55.8 & -51.7 \\
\hline $\mathrm{O}_{1}-\mathrm{C}_{6}-\mathrm{C}_{8}-\mathrm{O}_{14}$ & 175.1 & 175.8 & 22.9 & 29.3 & -29.8 & -35.7 & 156.8 & 153.3 & -29.6 & -42.4 & 158.0 & 157.1 & 61.5 & 65.7 \\
\hline $\mathrm{O}_{7}=\mathrm{C}_{6}-\mathrm{C}_{8}-\mathrm{H}_{9}$ & -122.5 & -122.7 & 80.4 & 86.0 & 36.8 & 32.2 & -144.0 & -148.7 & 34.1 & 22.4 & -142.1 & -144.3 & 123.2 & 129.1 \\
\hline $\mathrm{O}_{7}=\mathrm{C}_{6}-\mathrm{C}_{8}-\mathrm{C}_{10}$ & 118.2 & 117.3 & -37.2 & -33.0 & -81.2 & -87.0 & 97.3 & 91.7 & -83.3 & -96.5 & 96.6 & 93.6 & 3.2 & 8.1 \\
\hline $\mathrm{O}_{7}=\mathrm{C}_{6}-\mathrm{C}_{8}-\mathrm{O}_{14}$ & -4.7 & -4.6 & -160.1 & -154.0 & 152.8 & 147.8 & -25.1 & 91.7 & 152.9 & 141.1 & -25.1 & -27.6 & -119.5 & -113.6 \\
\hline $\mathrm{C}_{6}-\mathrm{C}_{8}-\mathrm{C}_{10}-\mathrm{H}_{11}$ & -59.0 & -58.0 & -64.8 & -62.8 & -63.4 & -62.7 & -61.1 & -60.7 & -64.5 & -63.3 & -57.6 & -58.6 & -59.3 & -58.5 \\
\hline $\mathrm{C}_{6}-\mathrm{C}_{8}-\mathrm{C}_{10}-\mathrm{H}_{12}$ & 62.4 & 63.3 & 55.8 & 57.5 & 57.4 & 57.9 & 60.0 & 60.2 & 56.6 & 58.0 & 63.4 & 61.9 & 60.7 & 61.4 \\
\hline $\mathrm{C}_{6}-\mathrm{C}_{8}-\mathrm{C}_{10}-\mathrm{H}_{13}$ & -177.7 & -176.5 & 176.0 & 178.0 & 177.4 & 178.0 & 179.2 & 179.7 & 175.8 & 177.0 & -176.2 & -177.3 & -179.9 & -179.3 \\
\hline $\mathrm{H}_{9}-\mathrm{C}_{8}-\mathrm{C}_{10}-\mathrm{H}_{11}$ & -177.0 & -176.6 & -179.7 & -179.3 & -179.3 & 61.0 & -178.5 & -178.8 & -179.1 & -179.3 & -179.4 & 178.8 & -178.3 & -179.0 \\
\hline $\mathrm{H}_{9}-\mathrm{C}_{8}-\mathrm{C}_{10}-\mathrm{H}_{12}$ & -55.7 & -55.4 & -59.2 & -59.0 & -58.5 & -59.1 & -57.4 & -57.8 & -58.0 & -58.3 & -58.4 & -60.6 & -58.3 & -59.1 \\
\hline $\mathrm{H}_{9}-\mathrm{C}_{8}-\mathrm{C}_{10}-\mathrm{H}_{13}$ & 64.3 & 64.8 & 61.1 & 61.5 & 61.4 & 61.0 & 61.8 & 61.6 & 61.1 & 60.9 & 62.0 & 60.1 & 61.1 & 60.2 \\
\hline $\mathrm{O}_{14}-\mathrm{C}_{8}-\mathrm{O}_{10}-\mathrm{H}_{11}$ & 63.3 & 63.5 & 60.7 & 60.8 & 63.2 & 62.7 & 58.1 & 57.2 & 57.7 & 56.7 & 62.9 & 61.4 & 59.9 & 58.9 \\
\hline $\mathrm{O}_{14}-\mathrm{C}_{8}-\mathrm{O}_{10}-\mathrm{H}_{12}$ & $2-175.3$ & -175.2 & -178.7 & -178.8 & -176.1 & -177.0 & 179.2 & 178.2 & 178.8 & 177.8 & -176.1 & -178.1 & 179.9 & 178.8 \\
\hline $\mathrm{O}_{14}-\mathrm{C}_{8}-\mathrm{O}_{10}-\mathrm{H}_{13}$ & $3 \quad-55.4$ & -55.0 & -58.5 & -58.4 & -56.1 & -56.6 & -61.5 & -62.3 & -62.0 & -63.0 & -55.7 & -57.3 & -60.7 & -61.9 \\
\hline
\end{tabular}


Table 2 (Continued)

\begin{tabular}{|c|c|c|c|c|c|c|c|c|c|c|c|c|c|c|}
\hline \multirow[t]{2}{*}{ Parameter } & \multicolumn{2}{|l|}{$S s C$} & \multicolumn{2}{|l|}{ GskC } & \multicolumn{2}{|l|}{$G^{\prime} s k^{\prime} C$} & \multicolumn{2}{|l|}{$A s C$} & \multicolumn{2}{|l|}{$A a C$} & \multicolumn{2}{|l|}{$A s T$} & \multicolumn{2}{|l|}{$A s k T$} \\
\hline & B3LYP & MP2 & B3LYP & MP2 & B3LYP & MP2 & B3LYP & MP2 & B3LYP & MP2 & B3LYP & MP2 & B3LYP & MP2 \\
\hline $\mathrm{C}_{6}-\mathrm{C}_{8}-\mathrm{O}_{14}-\mathrm{H}_{15}$ & 2.9 & 1.8 & -36.9 & -43.8 & 44.3 & 49.6 & 170.8 & 171.6 & 174.0 & 175.7 & 25.0 & 29.1 & 171.2 & 163.6 \\
\hline $\mathrm{H}_{9}-\mathrm{C}_{8}-\mathrm{C}_{14}-\mathrm{H}_{15}$ & 119.4 & 118.9 & 79.5 & 73.6 & 160.3 & 165.8 & -72.6 & -71.5 & -70.6 & -69.0 & 144.4 & 148.9 & -72.6 & -79.5 \\
\hline $\mathrm{C}_{10}-\mathrm{C}_{8}-\mathrm{O}_{14}-\mathrm{H}_{15}$ & -120.9 & -120.8 & -161.6 & -166.9 & -80.3 & -74.1 & 50.0 & 52.0 & 51.9 & 54.6 & -96.1 & -91.1 & 49.2 & 42.6 \\
\hline \multicolumn{15}{|c|}{ Rotational constants (MHz) } \\
\hline$A$ & 4849.3 & 4842.2 & 4706.1 & 4726.4 & 4262.3 & 4217.8 & 4912.7 & 4913.0 & 4330.8 & 4252.1 & 3707.1 & 3644.9 & 3707.1 & 3683.5 \\
\hline$B$ & 1852.3 & 1863.8 & 1925.9 & 1935.2 & 1901.9 & 1901.6 & 1766.4 & 1761.3 & 1883.4 & 1864.8 & 2313.0 & 2154.6 & 2313.0 & 2305.3 \\
\hline$C$ & 1456.3 & 1464.9 & 1438.2 & 1441.7 & 1588.4 & 1625.2 & 1493.8 & 1515.7 & 1589.0 & 1660.5 & 1595.7 & 1684.2 & 1595.7 & 1628.2 \\
\hline
\end{tabular}

${ }^{\mathrm{a}}$ See Fig. 1 for atom numbering.

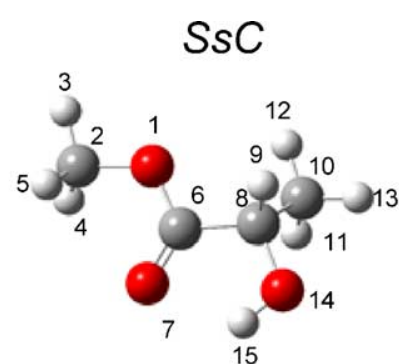

$\mathrm{O}=\mathrm{COC}:-0.2^{\circ} ; \mathrm{CCOH}: 2.9^{\circ}$; $\mathrm{O}=\mathrm{CCO}:-4.7^{\circ}$ $\mu=3.2 \mathrm{D}$

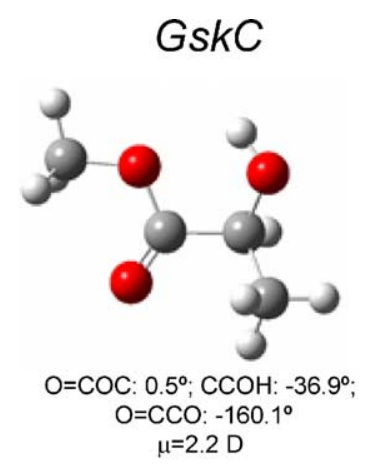

$-160.1^{\circ}$$$
\mu=2.2 \mathrm{D}
$$

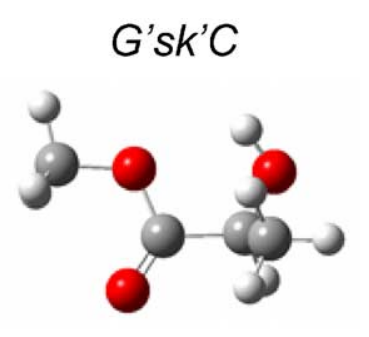

$\mathrm{O}=\mathrm{COC}:-0.7^{\circ} ; \mathrm{CCOH}: 44.3^{\circ}$; $\mathrm{O}=\mathrm{CCO}: 152.8^{\circ}$ $\mu=2.4 \mathrm{D}$
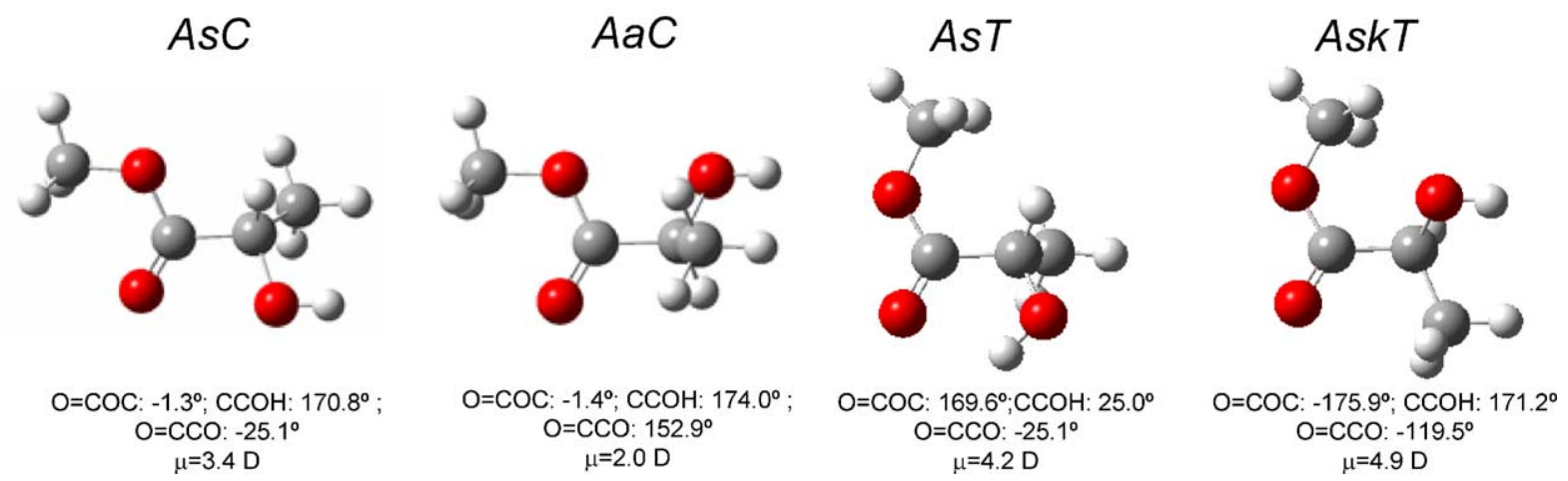

Fig. 1. Conformers of MLac with atom numbering scheme. For convenience, the values of the most relevant dihedral angles $\left({ }^{\circ}\right)$ and dipole moments $($ Debye) are included.

Table 3

DFT(B3LYP)/6-311++G(d,p) calculated relative energies $\left(\Delta E, \mathrm{~kJ} \mathrm{~mol}^{-1}\right)$ of the transition state structures (TSn) for interconversion between the cis conformers of $\mathrm{MLac}^{\mathrm{a}}$

\begin{tabular}{|c|c|c|c|c|c|}
\hline & $S s C$ & $G s k C$ & $G^{\prime} s k^{\prime} C$ & $A s C$ & $A a C$ \\
\hline \multicolumn{6}{|c|}{ Conformers } \\
\hline$S s C$ & - & $17.2(\mathrm{TS} 10)$ & $25.8(\mathrm{TS} 1)$ & 23.7 (TS4) & - \\
\hline & & $18.2(\mathrm{TS} 3)$ & 11.7 (TS9) & 21.9 (TS5) & \\
\hline GskC & $\begin{array}{c}9.6 \text { (TS10) } \\
10.6 \text { (TS3) }\end{array}$ & - & 1.6 (TS2) & - & 15.2 (TS7) \\
\hline$G^{\prime} s k^{\prime} C$ & $\begin{array}{l}17.3 \text { (TS1) } \\
19.9 \text { (TS9) }\end{array}$ & $1.0(\mathrm{TS} 2)$ & - & - & 13.6 (TS6) \\
\hline$A s C$ & $\begin{array}{l}2.3 \text { (TS4) } \\
0.4 \text { (TS5) }\end{array}$ & - & - & - & 1.2 (TS8) \\
\hline$A a C$ & - & 2.0 (TS7) & 0.9 (TS6) & 1.7 (TS8) & - \\
\hline
\end{tabular}

${ }^{\mathrm{a}} \Delta E$ correspond to the energy barriers from the bottom of the potential energy minima. 
was also found in the potential energy surface of MLac (AsT in Fig. 1) with relative energy of ca. $35 \mathrm{~kJ} \mathrm{~mol}^{-1}$ (Table 1).

Taking into consideration the calculated relative energies for the different conformers of MLac, their equilibrium populations in the gaseous phase at room temperature $(298 \mathrm{~K})$ were estimated using the Boltzmann distribution. The predicted populations ${ }^{2}$ for the three most stable conformers are 91.8, 4.6 and $3.5 \%$, respectively, while the remaining conformers have a total population of only ca. $0.1 \%$ and are of no practical interest.

The structural parameters for all the calculated conformers are provided in Table 2. In MLac, only the three most stable conformers are able to form internal H-bonds. The relative strengths of the H-bonds can be correlated with both the $\mathrm{O}-\mathrm{H}$ and $\mathrm{OH} \cdots \mathrm{O}$ distances. As expected, the $\mathrm{O}-\mathrm{H}$ distance is larger in $S s C(96.8 \mathrm{pm})$ than in both $G s k C$ and $G^{\prime} s k^{\prime} C(96.4 \mathrm{pm})$, while the $\mathrm{OH} \cdots \mathrm{O}=$ distance is considerably shorter in the first conformer $(208.5 \mathrm{pm})$ than the $\mathrm{OH} \cdots \mathrm{OCH}_{3}$ distances in $G s k C(215.4 \mathrm{pm})$ and $G^{\prime} s k^{\prime} C$ (222.4 pm), clearly demonstrating that the H-bond is stronger when it involves the carbonyl oxygen.

Internal rotations around the $\mathrm{C}-\mathrm{C}$ and $\mathrm{C}-\mathrm{O}(\mathrm{H})$ bonds allow the interconversion between all the conformers exhibiting a cis $\mathrm{O}=\mathrm{COC}$ axis. Energies of all the possible transition states interconnecting these conformers were calculated (Table 3). Fig. 2 depicts a "Cartesian" map with axes corresponding to the $\mathrm{O}=\mathrm{CCO}$ and $\mathrm{CCOH}$ dihedral angles, where the minima and transition states $(\mathrm{TS} n)$ were located. The map is analogous to that obtained for lactic acid [7], revealing that the replacement of the $\mathrm{OH}$ acid group by the $\mathrm{OCH}_{3}$ moiety does not lead to significant changes in the cis $\mathrm{C}=\mathrm{COC}$ potential energy surface. Four main pathways for conformational interconversion can be identified in the map, two corresponding essentially to changes in the conformation around the $\mathrm{C} \alpha-\mathrm{C}$ bond, and two to the rotation around the $\mathrm{C}-\mathrm{O}(\mathrm{H})$ bond. Rotation around the C-C bond defines the $S s C_{\leftrightarrow}^{\mathrm{TS} 1} G^{\prime} s k^{\prime} C_{\leftrightarrow}^{\mathrm{TS} 2} G s k C_{\leftrightarrow}^{\mathrm{TS} 3} S s C$ as well as the $A a C_{\leftrightarrow}^{\mathrm{TS} 8} A s C$ paths. The interconversions $S s C \leftrightarrow G s k C$ and $S s C \leftrightarrow G^{\prime} s k^{\prime} C$ can also pass through transition states TS10 and TS9, respectively. Rotation around the $\mathrm{C}-\mathrm{O}(\mathrm{H})$ bond defines the $A s C_{\leftrightarrow}^{\mathrm{TS} 4} S s C_{\leftrightarrow}^{\mathrm{TS} 5} A s C$ and $A a C_{\leftrightarrow}^{\mathrm{TS} 6} G^{\prime} s k^{\prime} C_{\leftrightarrow}^{\mathrm{TS} 2} G s k C_{\leftrightarrow}^{\mathrm{TS} 7} A a C$ paths.

Fig. 3 shows the approximate potential energy profiles for interconversion between the three most stable conformers of MLac. As for lactic acid [7], the energy barrier to convert the $G^{\prime} s k^{\prime} C$ conformer into the $G s k C$ form is low enough to be surpassed at the matrix temperature of $9 \mathrm{~K}$ [37], and the two conformers should then exist in the matrix in equilibrium. However, according to the calculations, at the cryogenic temperature of $9 \mathrm{~K}$, the population ratio $p_{\left(G^{\prime} k^{\prime} C\right)} / p_{(G s k C)}$ should be $\approx 2 \times 10^{-4}$. Thus, we can expect

\footnotetext{
${ }^{2}$ Calculations made based on DFT(B3LYP)/6-311++G(d,p) relative energies. Using MP2 relative energies, the estimated populations at room temperature are $\left(S s C: G s k C: G^{\prime} s k^{\prime} C\right) 84.6 \%: 8.4 \%: 6.9 \%$.
}

to observe experimentally only the $G s k C$ form, with a population equal to the sum of the populations of these two conformers existing in the vapor before deposition. Note that an increase in the temperature of the matrix up to $40 \mathrm{~K}$ does not lead to a significant change in the $p_{\left(G^{\prime} k^{\prime} C\right)} / p_{(G s k C)}$ population ratio, which remains smaller than ca. 0.1, thus precluding the observation of the $G^{\prime} s k^{\prime} C$ form within the range of experimental conditions used in this study.

\subsection{Vibrational spectra}

All conformers of MLac belong to the $\mathrm{C}_{1}$ point group, all their 39 vibrations being active in the infrared. The calculated vibrational spectra (B3LYP) for the five most stable conformers and definition of internal coordinates used in the normal mode analysis are provided in Tables S1-S6 (supplementary material). Vibrational assignments for the compound isolated in both argon and xenon matrices are displayed in Table 4.

The infrared spectra of matrix isolated MLac are presented in Fig. 4. In this figure, the experimental spectra (in argon) resulting from deposition of the vapor of the compound at two different temperatures (298 and $373 \mathrm{~K}$ ) are compared with the theoretically predicted spectra for conformers $S s C$ and $G s k C$. As expected considering the estimated relative populations of the conformers at the two temperatures used [298 K: $S s C, 91.8 \%, G s k C+G^{\prime} s k^{\prime} C$, $8.1 \%$; $\left.373 \mathrm{~K}: S s C, 85.6 \%, G s k C+G^{\prime} s k^{\prime} C, 14.2 \%\right]$, the bands corresponding to conformer $S s C$ largely dominate the experimental spectra. However, the most intense bands due to the less stable conformer can also be observed. These bands have higher relative intensities in the spectrum of a matrix obtained by deposition of MLac vapor at elevated temperature $(373 \mathrm{~K})$. The carbonyl stretching region of the experimental spectra is particularly useful for the identification of the less stable conformer, because the IR bands in this range are predicted to be intense and well separated (Table 4; see also Fig. 4 and Tables S2 and S3 (supplementary material)). In argon, the carbonyl stretching band of the most stable conformer is observed at $1746 \mathrm{~cm}^{-1}$ (calculated, $1747.4 \mathrm{~cm}^{-1}$ ), while that due to $G s k C$ appears as a site split doublet at $1765 / 1763 \mathrm{~cm}^{-1}$ (calculated $1771.9 \mathrm{~cm}^{-1}$ ). Other features ascribable to $G s k C$ are observed (in argon) at $3629 / 3626 \mathrm{~cm}^{-1}\left(v \mathrm{OH}\right.$, site splitted), $2860 \mathrm{~cm}^{-1}(v \mathrm{C}-\mathrm{H})$, $1368 \mathrm{~cm}^{-1}(\delta \mathrm{COH}), \quad 1327 \mathrm{~cm}^{-1}(\gamma \mathrm{C}-\mathrm{H}), \quad 1226 / 1224 /$ $1221 \mathrm{~cm}^{-1}\left(\nu \mathrm{C}-\mathrm{O}_{\text {ester }}\right.$; site splitted $), 1084 \mathrm{~cm}^{-1}\left(\gamma \mathrm{CH}_{3}{ }^{\prime}\right)$, $1052 \mathrm{~cm}^{-1}(v \mathrm{O}-\mathrm{C}), 1139 / 1134 / 1129 \mathrm{~cm}^{-1}\left(v \mathrm{C}-\mathrm{O}_{\text {alcohol }}\right.$, site splitted) and $831 / 827 \mathrm{~cm}^{-1}(\delta \mathrm{O}=\mathrm{C}-\mathrm{O}$; site splitted $)$, all in good agreement with the calculated data $(3649.7,2819.1$, 1373.3, 1328.8, 1214.6, 1080.7, 1041.0, 1131.7 and $827.0 \mathrm{~cm}^{-1}$, respectively). Each of those bands increases in intensity in the spectrum obtained from the vapor at higher temperature. In xenon, similar results were obtained, though the slightly larger average bandwidths make the assignments of the bands due to the minor form less certain. 


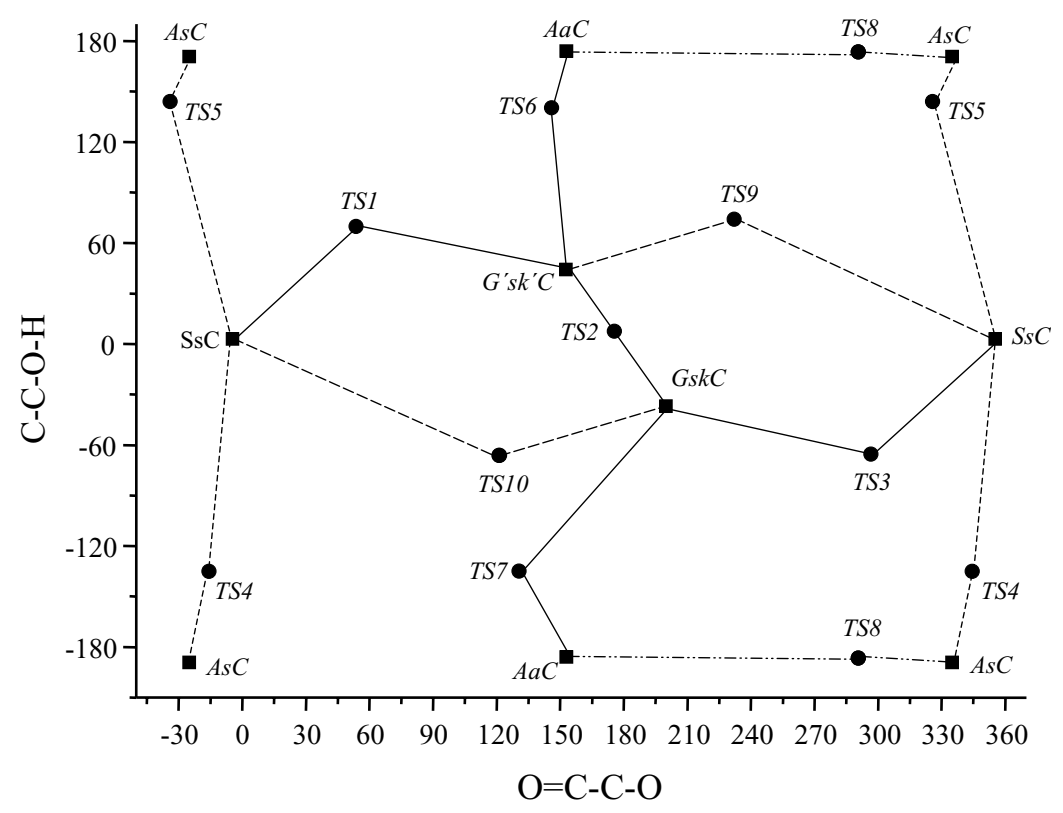

Fig. 2. Interconversion pathways among the whole set of cis $\mathrm{O}=\mathrm{COC}$ conformers of MLac.

The small band observed at $1779 \mathrm{~cm}^{-1}$ in the spectra of the compound in argon deserves here an additional comment. This band appears nearly at the predicted frequency for the carbonyl stretching mode in the ASC form $\left(1792 \mathrm{~cm}^{-1}\right.$; scaled value). However, the assignment of this band to the $A S C$ conformer seems very improbable, in view of its high relative energy (ca. $20 \mathrm{~kJ} \mathrm{~mol}^{-1}$ ). Its assignment to a combination tone of the most stable conformer appears more reasonable. Both $v(\mathrm{C} \alpha-\mathrm{C})+\gamma\left(\mathrm{CH}_{3}\right)^{\prime \prime}(638+1145)$ and $v(\mathrm{C}=\mathrm{O})+\tau(\mathrm{C} \alpha-\mathrm{C})(1746+41$, calculated scaled value) combinations are good candidates for being in the origin of the $1779 \mathrm{~cm}^{-1}$ band.
On the basis of the ratio of experimental intensities of the $\nu \mathrm{C}=\mathrm{O}$ bands assigned to the $S s C$ and to the $G s k C$ conformers, scaled by the ratio of the corresponding theoretical intensities (Tables S2 and S3 (supplementary material)), it was possible to approximately estimate the relative populations of these two forms in argon matrix. The obtained ratio SsC:GskC equal to 16.2 , in a matrix deposited from MLac vapor at $298 \mathrm{~K}$, correlates fairly well with the theoretical value (11.3; see Table 1).

After deposition, annealing experiments were performed in the xenon matrices, since the relatively low energy barriers for the $G s k C \rightarrow S s C$ isomerization $\left(\sim 10 \mathrm{~kJ} \mathrm{~mol}^{-1}\right.$;

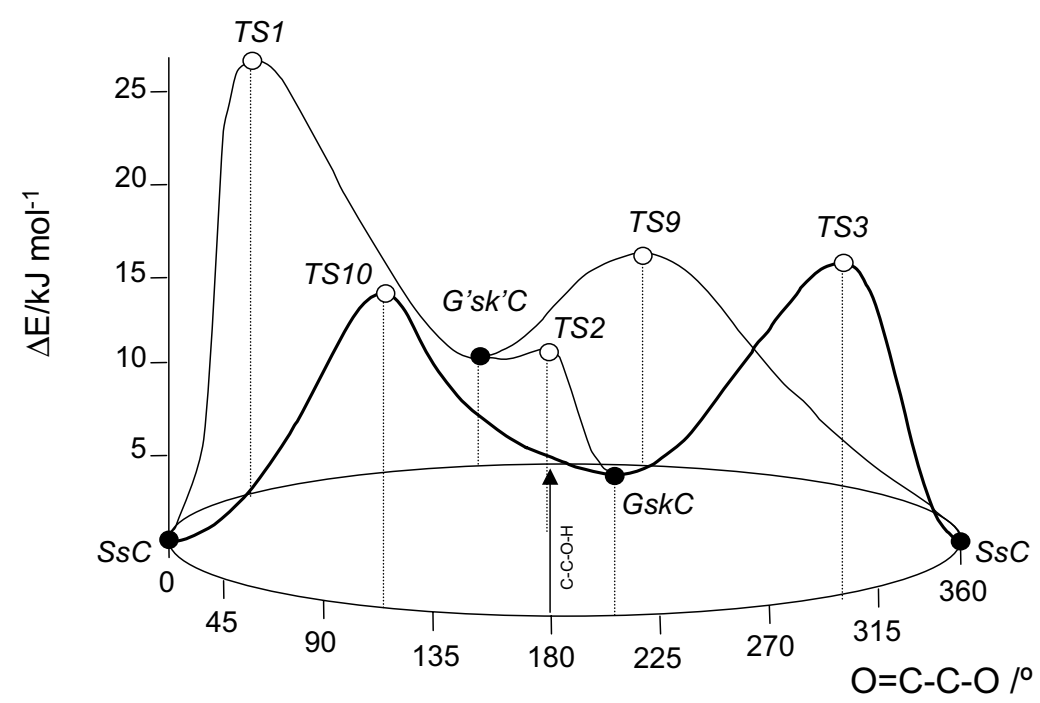

Fig. 3. DFT(B3LYP)/6-311++G(d,p) calculated potential energy profile for internal rotation around the $\mathrm{C} \alpha-\mathrm{C}$ and the $\mathrm{C}-\mathrm{O}(\mathrm{H})$ bonds for the SsC $\leftrightarrow G^{\prime} s k^{\prime} C \leftrightarrow G s k C \leftrightarrow S s C$ interconversions. 
Table 4

Observed frequencies for the $S s C$ and $G s k C$ conformers of MLac in argon and xenon matrices and calculated spectra

\begin{tabular}{|c|c|c|c|c|c|c|c|}
\hline \multirow[t]{2}{*}{$\begin{array}{l}\text { Approximate } \\
\text { description }^{\mathrm{a}}\end{array}$} & \multirow[t]{2}{*}{ Conformer } & \multicolumn{2}{|c|}{$\begin{array}{l}\text { Calculated values } \\
\text { DFT(B3LYP)/ } \\
6-311++G(d, p)\end{array}$} & \multicolumn{2}{|l|}{ Argon $(9 \mathrm{~K})$} & \multicolumn{2}{|c|}{ Xenon $(20 \mathrm{~K})$} \\
\hline & & $v\left(\mathrm{~cm}^{-1}\right)^{\mathrm{b}}$ & $I\left(\mathrm{~km} \mathrm{~mol}^{-1}\right)^{\mathrm{c}}$ & $v\left(\mathrm{~cm}^{-1}\right)$ & $I^{\mathrm{d}}$ & $v\left(\mathrm{~cm}^{-1}\right)$ & $I^{\mathrm{d}}$ \\
\hline \multirow[t]{2}{*}{$v(\mathrm{O}-\mathrm{H})$} & & 3569.6 & 81.7 & $3554 / 3549 / 3543 / 3540$ & $\mathrm{~m}$ & 3542 & $\mathrm{~m}$ \\
\hline & $G s k C$ & 3649.7 & 4.5 & $3629 / 3626$ & $\mathrm{w}$ & $3629 / 3621$ & $\mathrm{vw}$ \\
\hline$v\left(\mathrm{O}-\mathrm{CH}_{3}\right)_{\mathrm{as}}^{\prime}$ & & 3021.2 & 10.1 & 3040 & $\mathrm{vw}$ & 3033 & $\mathrm{vw}$ \\
\hline$v\left(\mathrm{O}-\mathrm{CH}_{3}\right)_{\mathrm{as}}^{\prime \prime}$ & & 2990.0 & 14.9 & $3012 / 3008$ & vw & \multirow{2}{*}{3000} & \\
\hline$v\left(\mathrm{CH}_{3}\right)_{\text {as }}^{\prime \prime}$ & & 2980.5 & 14.0 & 3000 & $\mathrm{vw}$ & & $\mathrm{VW}$ \\
\hline$v\left(\mathrm{CH}_{3}\right)_{\mathrm{as}}^{\prime}$ & & 2971.3 & 19.1 & 2992 & $\mathrm{w}$ & 2990 & $\mathrm{vw}$ \\
\hline$v\left(\mathrm{O}-\mathrm{CH}_{3}\right)_{\mathrm{s}}$ & & 2917.7 & 25.0 & 2965 & $\mathrm{w}$ & 2959 & $\mathrm{vw}$ \\
\hline$v\left(\mathrm{CH}_{3}\right)_{\mathrm{s}}$ & & 2902.4 & 13.4 & $2950 / 2946$ & vw & 2945 & $\mathrm{vw}$ \\
\hline \multirow[t]{2}{*}{$v(\mathrm{C}-\mathrm{H})$} & & 2870.7 & 21.1 & $2911 / 2897 / 2888$ & $\mathrm{w}$ & $2905 / 2883$ & $\mathrm{vw}$ \\
\hline & $G s k C$ & 2819.1 & 2.3 & 2860 & $\mathrm{w}$ & 2855 & - \\
\hline \multirow[t]{2}{*}{$v(\mathrm{C}=\mathrm{O})$} & & 1747.4 & 234.8 & 1746 & vs & 1741 & vs \\
\hline & GskC & 1771.9 & 17.9 & $1765 / 1763$ & $\mathrm{w}$ & $1756 / 1752$ & $\mathrm{w}$ \\
\hline$\delta\left(\mathrm{CH}_{3}\right)_{\mathrm{as}}^{\prime \prime}$ & & 1475.0 & 8.6 & \multirow{2}{*}{1463} & \multirow[b]{2}{*}{$\mathrm{m}$} & \multirow{3}{*}{1459} & \\
\hline$\delta\left(\mathrm{O}-\mathrm{CH}_{3}\right)_{\mathrm{as}}^{\prime}$ & & 1472.8 & 7.7 & & & & $\mathrm{~s}$ \\
\hline$\delta\left(\mathrm{CH}_{3}\right)_{\mathrm{as}}{ }^{\prime}$ & & 1468.7 & 10.9 & 1456 & $\mathrm{vw}$ & & \\
\hline$\delta\left(\mathrm{O}-\mathrm{CH}_{3}\right)_{\mathrm{as}}{ }^{\prime \prime}$ & & 1460.6 & 9.1 & $1450 / 1449$ & $\mathrm{w}$ & 1448 & $\mathrm{w}$ \\
\hline$\delta\left(\mathrm{O}-\mathrm{CH}_{3}\right)_{\mathrm{s}}$ & & 1448.8 & 7.0 & 1440 & $\mathrm{w}$ & 1438 & $\mathrm{w}$ \\
\hline \multirow[t]{2}{*}{$\delta(\mathrm{COH})$} & & 1397.7 & 10.2 & $1412 / 1404$ & vw & 1410 & $\mathrm{vw}$ \\
\hline & $G s k C$ & 1373.3 & 3.4 & 1368 & $\mathrm{w}$ & 1367 & $\mathrm{w}$ \\
\hline$\delta\left(\mathrm{CH}_{3}\right)_{\mathrm{s}}$ & & 1381.2 & 7.9 & 1374 & $\mathrm{vw}$ & 1371 & $\mathrm{vw}$ \\
\hline \multirow[t]{2}{*}{$\gamma(\mathrm{C}-\mathrm{H})$} & & 1326.6 & 1.0 & 1323 & $\mathrm{w}$ & 1326 & $\mathrm{w}$ \\
\hline & $G s k C$ & 1328.8 & 1.4 & 1327 & $\mathrm{w}$ & 1339 & \\
\hline \multirow[t]{2}{*}{$v(\mathrm{C}-\mathrm{O})_{\text {ester }}$} & & 1257.8 & 226.9 & 1266 & vs & 1264 & vs \\
\hline & GskC & 1214.6 & 29.5 & $1226 / 1224 / 1221$ & $\mathrm{~m}$ & $1218 / 1207$ & $\mathrm{~m}$ \\
\hline$\delta(\mathrm{CCH})$ & & 1226.8 & 138.8 & $1247 / 1244 / 1238 / 1235$ & $\mathrm{~s}$ & 1236 & $\mathrm{~s}$ \\
\hline$\gamma\left(\mathrm{O}-\mathrm{CH}_{3}\right)^{\prime}$ & & 1187.9 & 1.5 & 1192 & vw & 1189 & vw \\
\hline$\gamma\left(\mathrm{O}-\mathrm{CH}_{3}\right)^{\prime \prime}$ & & 1152.6 & 1.6 & 1159 & - & - & - \\
\hline$\gamma\left(\mathrm{CH}_{3}\right)^{\prime \prime}$ & & 1138.5 & 159.8 & $1149 / 1145 / 1143$ & $\mathrm{~s}$ & $1150 / 1141$ & $\mathrm{~s}$ \\
\hline \multirow[t]{2}{*}{$\gamma\left(\mathrm{CH}_{3}\right)^{\prime}$} & & 1089.6 & 25.4 & 1097 & $\mathrm{w}$ & 1096 & $\mathrm{vw}$ \\
\hline & $G s k C$ & 1080.7 & 1.2 & 1084 & $\mathrm{vw}$ & 1081 & $\mathrm{vw}$ \\
\hline$v(\mathrm{C}-\mathrm{C})$ & & 1032.5 & 36.8 & 1040 & $\mathrm{~m}$ & 1040 & $\mathrm{~m}$ \\
\hline \multirow[t]{2}{*}{$v(\mathrm{O}-\mathrm{C})$} & & 978.4 & 16.6 & 989/987 & w & $985 / 979$ & $\mathrm{w}$ \\
\hline & $G s k C$ & 1041.0 & 4.8 & 1052 & $\mathrm{w}$ & 1047 & $\mathrm{w}$ \\
\hline \multirow[t]{2}{*}{$v(\mathrm{C}-\mathrm{O})_{\mathrm{al} .}$} & & 915.1 & 7.0 & 926 & w & 924 & $\mathrm{w}$ \\
\hline & GskC & 1131.7 & 6.0 & $1139 / 1134 / 1129$ & $\mathrm{w}$ & - & \\
\hline \multirow[t]{2}{*}{$\delta(\mathrm{O}=\mathrm{C}-\mathrm{O})$} & & 838.7 & 6.2 & 844 & $\mathrm{vw}$ & 844 & $\mathrm{vw}$ \\
\hline & $G s k C$ & 827.0 & 1.6 & $831 / 827$ & $\mathrm{vw}$ & $833 / 828$ & $\mathrm{vw}$ \\
\hline$\gamma(\mathrm{C}=\mathrm{O})$ & & 750.2 & 9.3 & 758 & $\mathrm{vw}$ & 757 & $\mathrm{vw}$ \\
\hline$v\left(\mathrm{C}_{\alpha}-\mathrm{C}\right)$ & & 637.5 & 13.1 & $654 / 638 / 631 / 623$ & vw & $653 / 636$ & vw \\
\hline
\end{tabular}

${ }^{\mathrm{a}}$ See Table S1 (supplementary material) for definition of coordinates. $v$, bond stretching; $\delta$, bending; $\gamma$, rocking; $\tau$, torsion; s, symmetric; as, asymmetric.

${ }^{\mathrm{b}}$ Scale factors $=0.955$ for frequencies above $2800 \mathrm{~cm}^{-1}$, and 0.984 for frequencies below $2800 \mathrm{~cm}^{-1}$.

${ }^{\mathrm{c}}$ Intensities were weighted by the population factor $(T=298 \mathrm{~K})$ for each conformer as predicted by the DFT(B3LYP)/6-311++G(d,p): 0.981 for $S s C$, and 0.081 for $G s k C$, that also includes the population of the $G^{\prime} s k^{\prime} C$ conformer as explained in the text.

d s, strong; w, weak; vw, very weak; vs, very strong; m, medium.

Table 3) can, in principle, make possible the observation of this process upon warming of these matrices. According to the Barnes relationship [37], for such energy barrier, the conversion can be expected to start only at $\sim 37 \mathrm{~K}$.
Note that at this temperature, argon matrices are too much soft to avoid extensive aggregation. However, for the studied compound, aggregation was also found to be significant in the xenon matrices at $37 \mathrm{~K}$, thus preventing 

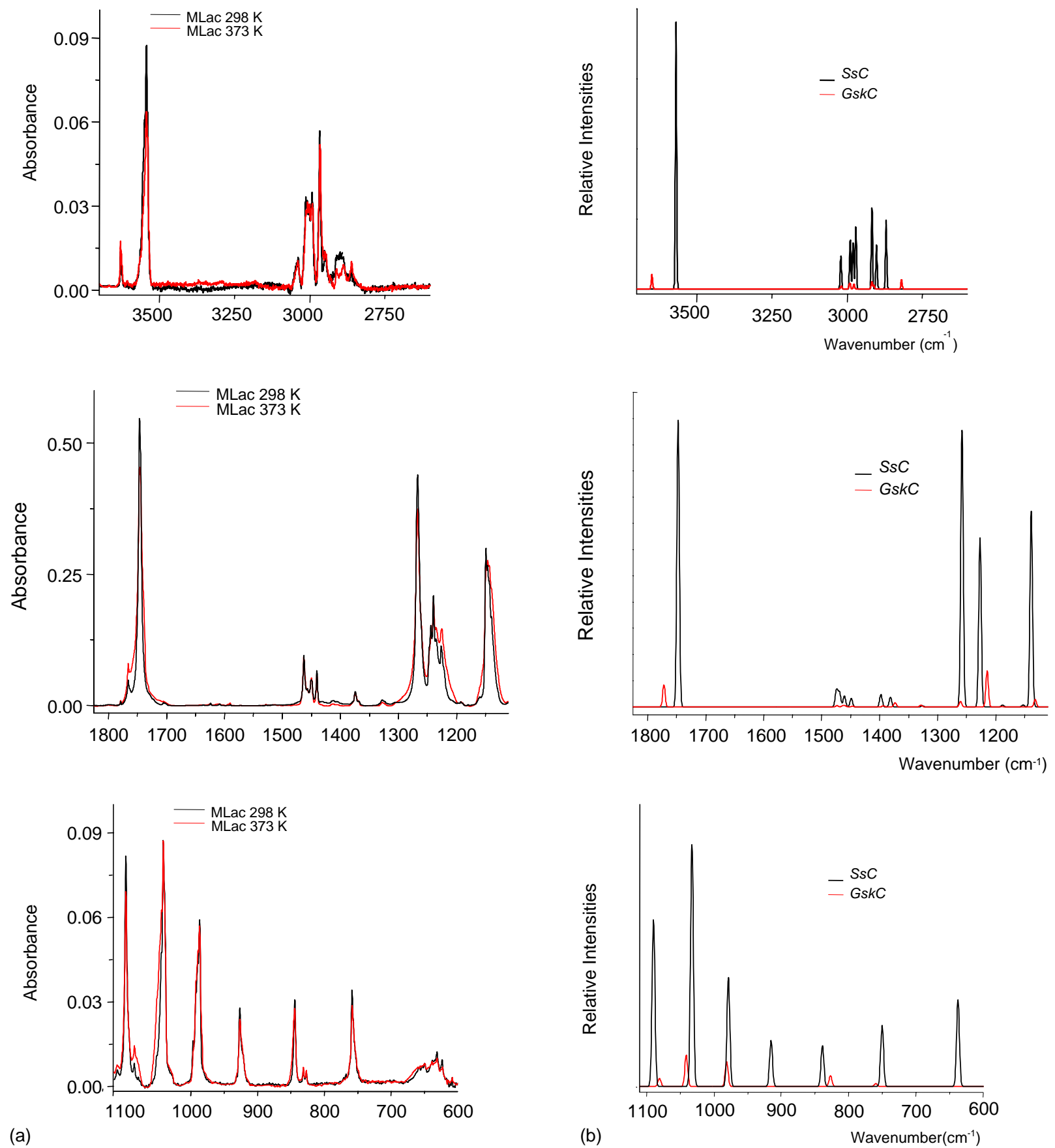

Fig. 4. Infrared spectra of MLac: (a) trapped in an argon matrix obtained immediately after deposition at $9 \mathrm{~K}$; (b) calculated spectra for the $S s C$ and $G s k C$ conformers (relative intensities scaled by the calculated relative populations at $298 \mathrm{~K}$ ).

unequivocal observation of the putative conformational isomerization.

\section{Conclusion}

Matrix-isolation FTIR spectroscopy, supported by DFT(B3LYP)/6-311++G(d,p) and MP2/6-31++G(d,p) calculations, allowed unequivocal observation and spectral signature characterization of the two most stable conformers of methyl lactate.
Assignment of the observed spectra was carried out on the basis of comparison with the theoretical spectra and gas-phase thermal population of the less stable conformers prior to deposition.

In consonance with the theoretical predictions, conformer SsC represents the most stable form of the compound in the gaseous phase, being the dominant species trapped in both argon and xenon matrices, while the contribution of $G s k C$ conformer to the observed spectra is small, though clearly discernible. The third most stable conformer of MLac, 
$G^{\prime} s k^{\prime} C$, could not be observed in the matrices, since this form can convert quantitatively to the relatively more stable $G s k C$ conformer at the matrix temperatures, due to the very low energy barrier separating these two conformers $\left(\approx 1 \mathrm{~kJ} \mathrm{~mol}^{-1}\right)$.

\section{Acknowledgements}

This work was supported by the Portuguese Fundação para a Ciência e a Tecnologia (Research Project POCTI/ QUI/43366/2001 and Grant FCT \#SFRH/BPD/11499/2002) and FEDER.

\section{References}

[1] E. Scott, J. Am. Acad. Derm. 11 (1984) 867.

[2] Y. Guzel, J. Mol. Struct. (Theochem) 366 (1996) 131.

[3] L. Moy, H. Murad, R. Moy, J. Derm. Surg. Oncol. 19 (1996) 243.

[4] E. Scott, Can. J. Derm. 43 (1989) 222.

[5] M. Kleerebezem, J. Hugenholtz, Curr. Opin. Biotechnol. 14 (2003) 232.

[6] D. Gilding, M. Reed, Polymers 20 (1979) 1459.

[7] A. Borba, A. Gómez-Zavaglia, L. Lapinski, R. Fausto. Rotational isomers of lactic acid: first experimental observation of higher energy forms, Phys. Chem. Chem. Phys. 6 (2004) published as advanced articles: doi: 10.1039/b316642b.

[8] C. Blom, A. Bauder, J. Am. Chem. Soc. 104 (1982) 2993.

[9] H. Hasegawa, O. Ohashi, I. Yamaguchi, J. Mol. Struct. 82 (1982) 205.

[10] H. Hollenstein, T. Ha, H.s. Günthard, J. Mol. Struct. 146 (1986) 289.

[11] K. Iijima, M. Kato, B. Beagley, J. Mol. Struct. 295 (1993) 289.

[12] S. Jarmelo, R. Fausto, Phys. Chem. Chem. Phys. 4 (2002) 1555.

[13] S. Jarmelo, T. Maria, M. Leitão, R. Fausto, Phys. Chem. Chem. Phys. 2 (2000) 1155.

[14] S. Jarmelo, T. Maria, M. Leitão, R. Fausto, Phys. Chem. Chem. Phys. 3 (2001) 387

[15] S. Jarmelo, R. Fausto, J. Mol. Struct. 509 (1999) 183.

[16] H. Hollenstein, R. Schar, N. Schwizgebel, G. Grassi, Hs. Günthard, Spectrochim. Acta A 39 (1983) 193.
[17] Y. Nakao, H. Sugeta, Y. Kyoguku, Chem. Lett. 4 (1984) 623.

[18] N. Borho, M.A. Suhm. Tailor-made aggregates of $\alpha$-hydroxy esters in supersonic jets, Phys. Chem. Chem. Phys. (2004) doi: 10.1039/ b315765b.

[19] I. Reva, S. Stepanian, L. Adamowicz, R. Fausto, Chem. Phys. Lett. 374 (2003) 631.

[20] A. Gómez-Zavaglia, R. Fausto, Phys. Chem. Chem. Phys. 5 (2003) 52 .

[21] R. Bursi, F.J. Devlin, P. Stephens, J. Am. Chem. Soc. 112 (1990) 9431

[22] W. Coleman, B. Gordon, Appl. Spectrosc. 43 (1989) 305.

[23] G. Cassanas, M. Morssli, E. Fabrègue, L. Bardet, J. Raman Spectrosc. 22 (1991) 409.

[24] D. Gigante, F. Long, L. Bodack, J. Evans, J. Kallmerten, L. Nafie, T. Freedman, J. Phys. Chem. A 103 (1999) 1523.

[25] T. Freeman, E. Lee, L. Nafie, J. Phys. Chem. A 104 (2000) 3944.

[26] N. Borho, M. Suhm, Org. Biomol. Chem. 1 (2003) 4351.

[27] T. Häber, U. Schmitt, M. Suhm, Phys. Chem. Chem. Phys. 1 (1999) 5573.

[28] M. Frisch, G. Trucks, H. Schlegel, G. Scuseria, M. Robb, J. Cheeseman, V. Zakrzewski, J. Montgomery, R. Stratmann, K. Burant, S. Dapprich, J. Millam, A. Daniels, K. Kudin, M. Strain, O. Farkas, J. Tomasi, V. Barone, M. Cossi, R. Cammi, B. Mennucci, C. Pomelli, C. Adamo, S. Clifford, J. Ochterski, G. Petersson, P. Ayala, Q. Cui, K. Morokuma, D. Malick, A. Rabuck, K. Raghavachari, J. Foresman, J. Cioslowski, J. Ortiz, A. Baboul, B. Stefanov, G. Liu, A. Liashenko, P. Piskorz, I. Komaromi, R. Gomperts, R. Martin, D. Fox, T. Keith, M. Al-Laham, C. Peng, A. Nanayakkara, M. Challacombe, P. Gill, B. Johnson, W. Chen, M. Wong, J. Andres, C. Gonzalez, M. HeadGordon, E.S. Replogle, J. Pople, Gaussian 98, revision A.9, Gaussian Inc., Pittsburgh, PA, 1998.

[29] M. Frisch, M. Head-Gordon, J. Pople, Chem. Phys. Lett. 166 (1990) 281.

[30] A. Becke, Phys. Rev. A 38 (1988) 3098.

[31] C. Lee, W. Yang, R. Parr, Phys. Rev. B 37 (1988) 785.

[32] S. Vosko, L. Wilk, M. Nusair, Can. J. Phys. 58 (1980) 1200.

[33] P. Csaszar, P. Pulay, J. Mol. Struct. (Theochem) 114 (1984) 31.

[34] J. Schachtschneider, Technical Report Shell Development Co., Emeryville, CA, 1969.

[35] C. Peng, H. Schlegel, Israel J. Chem. 33 (1994) 449.

[36] I. Reva, S. Stepanian, L. Adamowicz, R. Fausto, J. Phys. Chem. A 105 (2001) 4773.

[37] A. Barnes, J. Mol. Struct. 113 (1984) 161. 